\title{
A Rare Presentation of Idiopathic Multiple Impacted Teeth and Its Management with Multiunit Abutment and Malo Bridge
}

\author{
Rakshith Hegde ${ }^{1}$, Nivya John², Athma Shetty ${ }^{3}$, Manoj Shetty ${ }^{4}$, Nitish Garlapati ${ }^{5}$, Sushmitha Palaniswamy ${ }^{6}$
}

\begin{abstract}
Aim and objective: This case report aims to bring into limelight the full mouth rehabilitation in the non-syndromic presentation of multiple carious teeth and impacted teeth, using multiunit abutments.

Background: Presentation of multiple impacted teeth is very rare among the Indian population unless it is associated with syndromes or eruption disturbances. However, there are very rare instances where such patients do present with non-syndromic associations. Functional and esthetic rehabilitation of the condition becomes very crucial especially in young patients. Implants by far are considered to provide the best definitive option.

Materials and methods: The surgical protocol was to extract existing partially erupted teeth and immediately place implants. Multiunit screwretained abutments were loaded within 6 weeks. A passively seating full-arch prosthesis was designed with anterior Malo bridge. This was done to mask the labially facing unesthetic screw holes of the angulated abutments.

Conclusion: In the above-mentioned case, delivery of a functional prosthesis was possible because of the use of multiunit abutments. Furthermore, the Malo bridge masked the labially facing unesthetic screw holes of the angulated abutments, thereby providing good esthetic results. Additionally, there is also the option of prosthesis retrievability, wherein the practitioner can unscrew the prosthesis whenever a surgical removal of the impacted teeth is necessary.

Clinical significance: With the use of multiunit abutments, it is possible to deliver fast treatment without compromising on esthetics and functionality.

Keywords: Full mouth rehabilitation, Implants, Multiple impacted teeth, Multiunit abutments.

World Journal of Dentistry (2021): 10.5005/jp-journals-10015-1869
\end{abstract}

\section{INTRODUCTION}

An impacted tooth is a common presentation among people; however, multiple impacted teeth usually are rare and often associated with syndromes such as cleidocranial dysplasia, Gardner's syndrome, Down's syndrome, and Noonan's syndrome. ${ }^{1}$ Nevertheless, non-syndromic associated multiple impacted teeth can present due to various causes such as local biomechanical inhibitions, insufficient maxillofacial skeletal development, or eruption disturbances. ${ }^{2}$ These patients usually present with no other systemic conditions.

Impaction of multiple permanent teeth is a rare condition especially with no syndromic associations, and the only handful of cases have been reported in the literature. ${ }^{1,3-6}$ Conventionally, the treatment plan for such cases includes the extraction of the teeth and provide a removable prosthesis. The authors Ghaeminia et al. revealed in Cochrane's study that asymptomatic disease-free teeth can be retained and checked frequently at follow-up visits. ${ }^{7}$ Thus in this case, asymptomatic impacted teeth were retained and only the grossly decayed erupted teeth were extracted. Presentations like these are often treated with removable prosthesis. This case report is unique as it discusses the treatment plan of a patient with multiple impacted teeth, rehabilitated with implants. The prosthetic phase commenced after 6 weeks postimplant placement and a fixed implant-supported screw-retained prosthesis was delivered in the final prosthetic phase. This enabled the patient to lead a socially confident life.
${ }^{1-3}$ Department of Prosthodontics and Oral Implantology, AB Shetty Memorial Institute of Dental Sciences, NITTE (Deemed to be University), Mangaluru, Karnataka, India

${ }^{4}$ Department of Oral Implantology, $A B$ Shetty Memorial Institute of Dental Sciences, NITTE (Deemed to be University), Mangaluru, Karnataka, India

${ }^{5,6}$ Department of Prosthodontics and Oral Implantology, AB Shetty Memorial Institute of Dental Sciences, NITTE (Deemed to be University), Mangaluru, Karnataka, India

Corresponding Author: Nivya John, Department of Prosthodontics and Oral Implantology, $A B$ Shetty Memorial Institute of Dental Sciences, NITTE (Deemed to be University), Mangaluru, Karnataka, India, Phone: +91 9742024358, e-mail: nivyajohn@nitte.edu.in

How to cite this article: Hegde R, John N, Shetty A, et al. A Rare Presentation of Idiopathic Multiple Impacted Teeth and Its Management with Multiunit Abutment and Malo Bridge. World J Dent 2021;12(6):492-495.

Source of support: Nil

Conflict of interest: None

\section{Case Description}

A 24-year-old female patient visited the Department of Oral Implantology with a chief complaint of missing teeth and grossly decayed teeth for 10 years.

(-) The Author(s). 2021 Open Access This article is distributed under the terms of the Creative Commons Attribution 4.0 International License (https://creativecommons. org/licenses/by-nc/4.0/), which permits unrestricted use, distribution, and non-commercial reproduction in any medium, provided you give appropriate credit to the original author(s) and the source, provide a link to the Creative Commons license, and indicate if changes were made. The Creative Commons Public Domain Dedication waiver (http://creativecommons.org/publicdomain/zero/1.0/) applies to the data made available in this article, unless otherwise stated. 


\section{History of Presenting Illness}

The patient noticed that the teeth stopped erupting at the age of 10 years.

\section{Dental History}

The patient visited the dental clinic for temporary restorations, her orthodontic history revealed that surgical extrusion and orthodontic movement would not provide a predictable result due to the unfavorable positions and possible ankylosis of the impacted teeth.

\section{General Examination}

Her medical history revealed ideal birth conditions (3.5 kg birth weight, 38 weeks). No trauma during childhood. The patient reported no medical and family history suggestive of this condition. No consanguineous marriage was reported. General physical examination was not suggestive of any syndromes either.

\section{Clinical Examination}

The patient was well built with $148 \mathrm{~cm}$ height and $48 \mathrm{~kg}$. No other aberrations were observed. Routine blood investigations revealed normal INR, bleeding time, and clotting time. Glycated hemoglobin was $5.2 \mathrm{mg} / \mathrm{dL}$. She had no history of any medications.

Extraoral examination of the face revealed a concave profile and collapsed smile due to loss of vertical dimension on the right side.

Intraoral examination revealed several isolated areas of edentulousness with grossly decayed dentition. Soft tissue was pink and healthy.

\section{Radiological Evaluation}

Orthopantomography (OPG) revealed multiple impacted teeth, i.e., in the maxillary arch (FDI): 18, 17, 14, 13, 23, 24, 26, 27, 28 and in the mandibular arch: 48, 47, 46, 33, 34, 36, 37, 38 (Fig. 1). Cone-beam CT was taken to evaluate the three-dimensional position of the teeth and also measure the width of the bone (Fig. 2).

\section{Treatment Planning}

The following teeth were planned to be extracted during the surgical phase (FDI). Maxillary arch: 16, 15, 12, 11, 21, 22, 62, 63, and 25 and in the mandibular arch: 45, 44, 83, 41, 42, 31, 32, 73, and 35 SLA implants (Ankylos) were selected as per the available bone width and height.
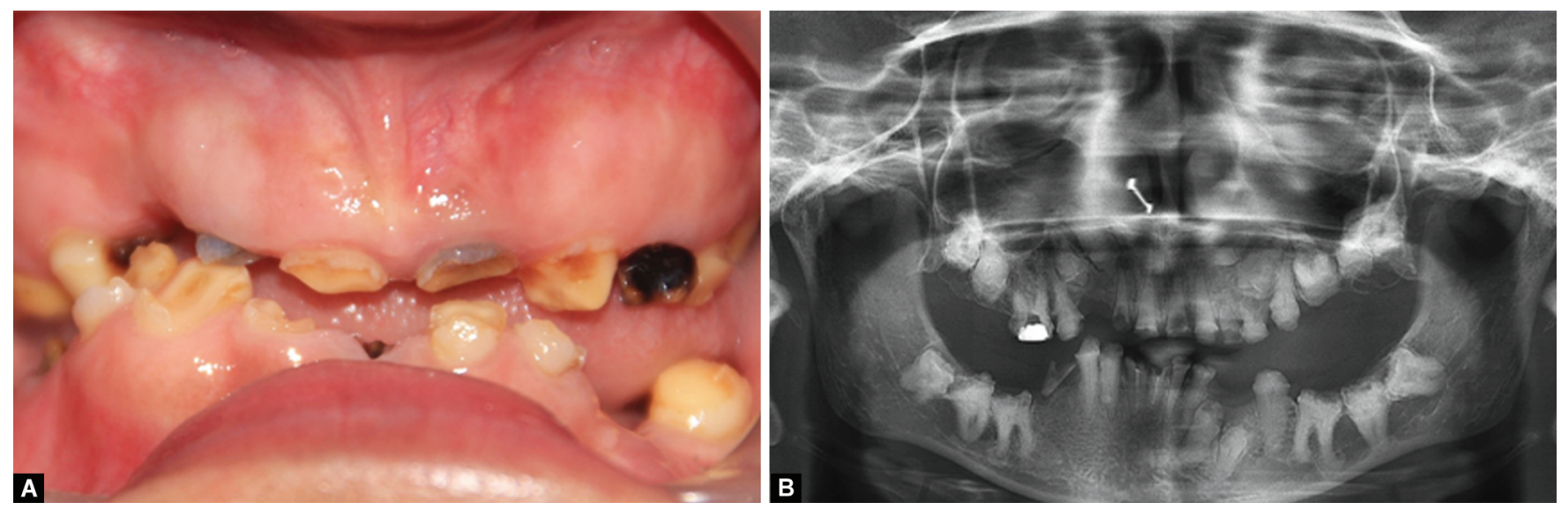

\section{Surgical Procedure}

The patient was prescribed antibiotics (amoxicillin $500 \mathrm{mg}$ ) as per Misch's protocol. ${ }^{8}$ Long-acting local anesthetic (lidocaine) was administered at the site. Extractions were carried out as atraumatically as possible. The corrugated bony architecture was smoothened and the bone shavings collected during the procedure were mixed with a corticocancellous allograft (Rocky mountain $0.5 \mathrm{cc}$ ) and used for lateral augmentation. Four implants (A9.5 and A11) each were placed in the maxillary and mandibular ridge. ${ }^{9,10}$ An immediate postoperative OPG showed the parallel placement of the implants in the maxillary. In the mandibular ridge, the distal implant of the right side was angulated to get a larger anteroposterior spread (Fig. 3). Sutures were placed (Vicryl 3.0) and patient was recalled after 6 weeks for prosthetic phase. Patient was advised to use antibiotics and analgesics (NSAIDs) and oral antibacterial mouth rinses (chlorhexidine gluconate) for a week.

\section{Prosthetic Phase}

Prosthetic procedures commenced after 6 weeks of soft and hard tissue healing. The patient was recalled and the closure caps were removed non-invasively. Impressions and maxilla mandibular relations were recorded with temporary abutments. Sulcus formers were placed for 2 weeks during the transition phase. During the definitive phase, multiunit abutments were torqued to $25 \mathrm{~N} \mathrm{~cm}$ (Fig. 4). ${ }^{10,11}$ The cast models and impressions were sent to the laboratory for designing and casting. A full mouth metal trial was done and clearance of $2 \mathrm{~mm}$ was ensured. A hybrid prosthesis called a "Malo Bridge" was designed for the patient considering that screw holes may pose esthetic challenges (Fig. 5). ${ }^{12,13}$ The final full-arch prosthesis was verified with a one-screw test for passive fitting. The prosthesis screws were tightened following the manufacturer's instructions to $15 \mathrm{~N} \mathrm{~cm}$. Teflon tape was used to obliterate the screw channels. Occlusal components like interocclusal contacts, eccentric contacts were modified as per rules of implant biomechanics (Misch) phonetics and esthetics were verified (Fig. 6).

\section{Postoperative Instructions}

Oral hygiene instructions were given. A Waterpik plaque removal kit was prescribed, and oral mouth rinses were provided.

Figs 1A and B: (A) Preoperative smile view; (B) Preoperative OPG 


\section{Follow-up}

Patient was recalled after 6 months and 1 year post-cementation. The OPGs revealed stable crestal levels. Clinically, the prosthesis maintained ideal soft tissue conditions (Fig. 7).

\section{Discussion}

Raghoebar (1989) described impaction as the cessation of an eruption of the tooth due to a physical barrier. It could be syndromic,

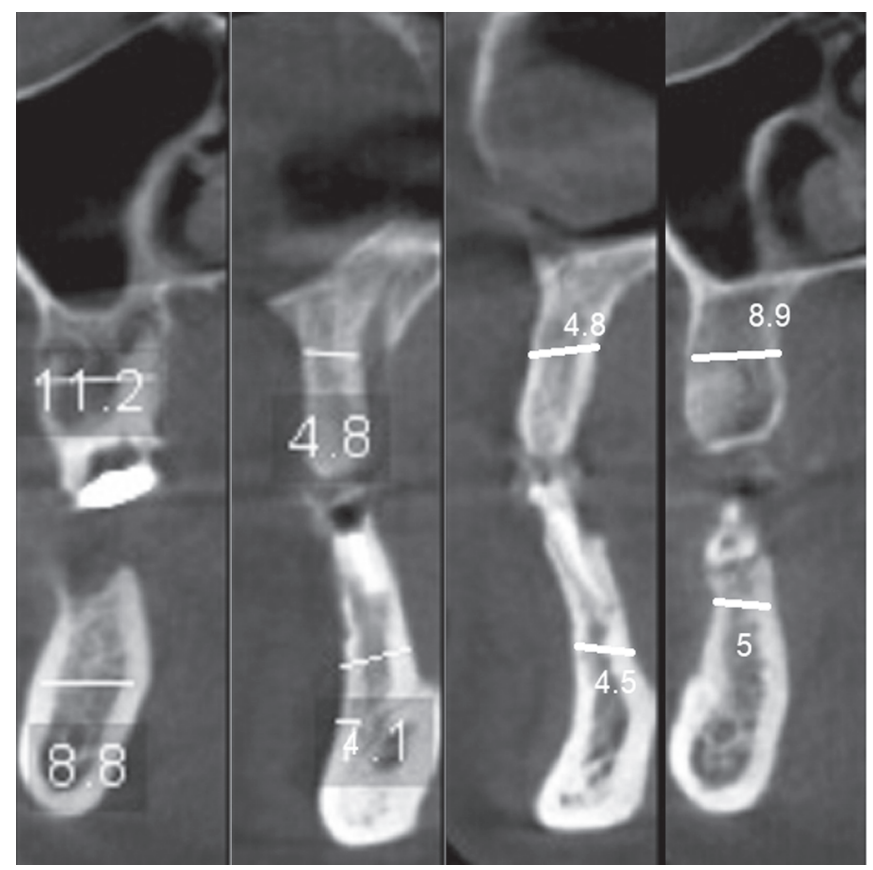

Fig. 2: CBCT sections of 16, 12, 22, 25 and 35, 43, 44, 45 familial, or even caused due to disturbances in eruption. Very rarely non-syndromic variants also arise which do not have any known metabolic and hormonal disorders. ${ }^{14-16}$ This case report presents a 24-year-old female patient with multiple impacted teeth. Orthodontic extrusions were contraindicated because of possible ankylosis of the teeth. Thus, extraction was indicated with respect to the erupted teeth that were mobile and carious. ${ }^{4}$

Implants were placed in the extraction sites along with lateral contour augmentation so that inevitable bundle bone loss can be compensated. Distal most implant in the mandibular ridge was angulated to create a larger anterior-posterior spread. The prosthetic phase included the use of a screw-retained multiunit prosthesis for a passive fit and correction of angulations. The added advantage of the screw-retained prosthesis is that, if the impacted teeth were to show signs of disease, the prosthesis could be retrieved easily and surgical removal can be done, as opposed to cement-retained prosthesis where the entire prosthesis has to be destroyed. The multiunit abutments also maintained a good hemidesmosome attachment, thus protecting the soft tissue

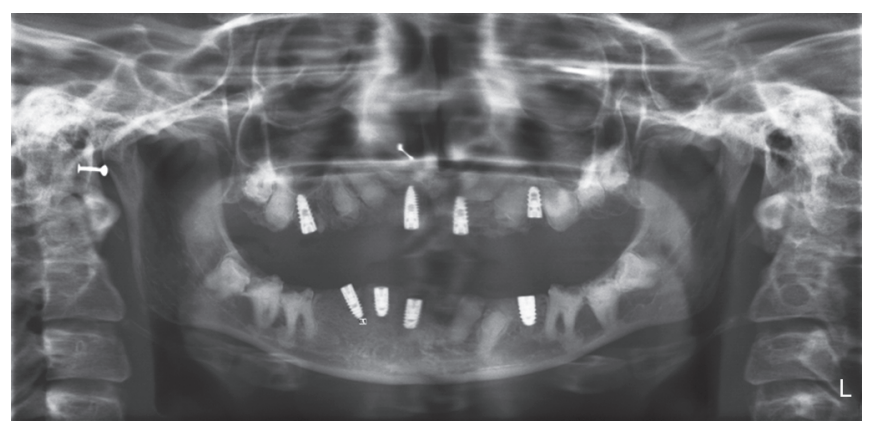

Fig. 3: Immediate postoperative X-ray
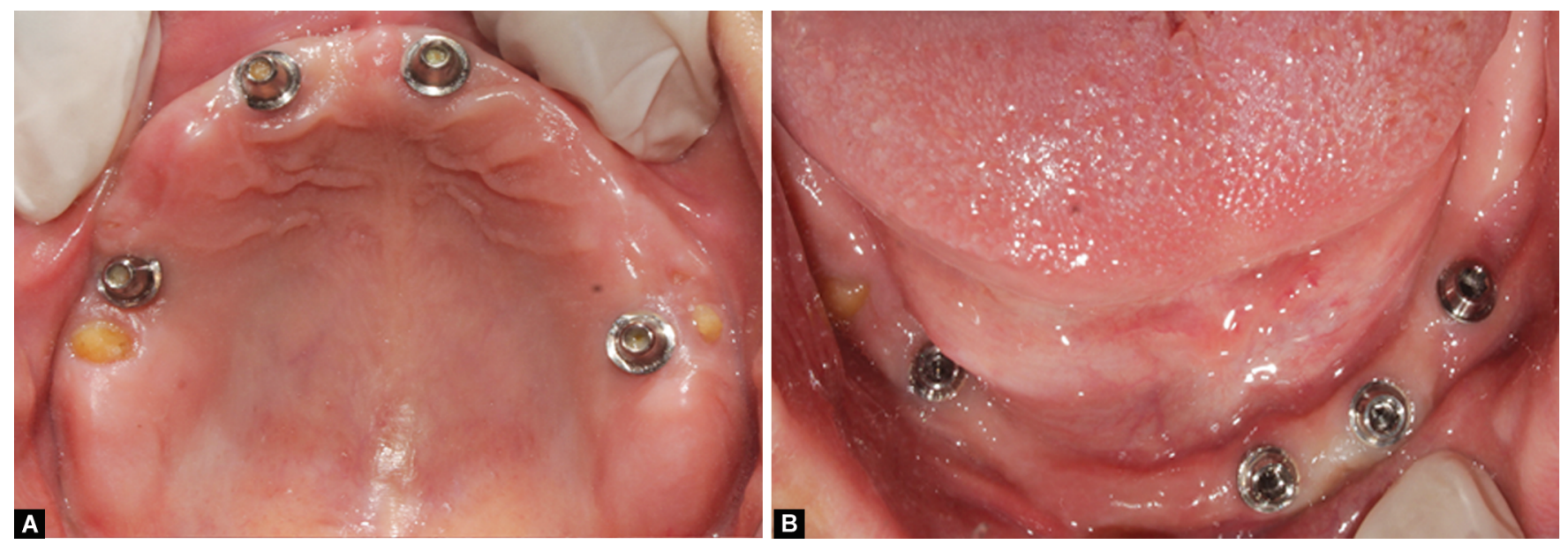

Figs $4 \mathrm{~A}$ and B: Multiunit abutment placement in the maxilla and mandible
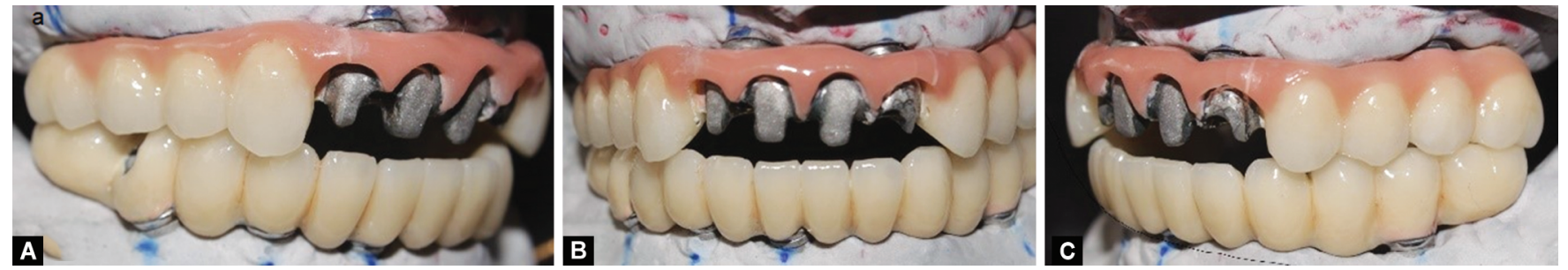

Figs 5A to C: Right lateral view (right), facial view (center), left lateral view (left) 

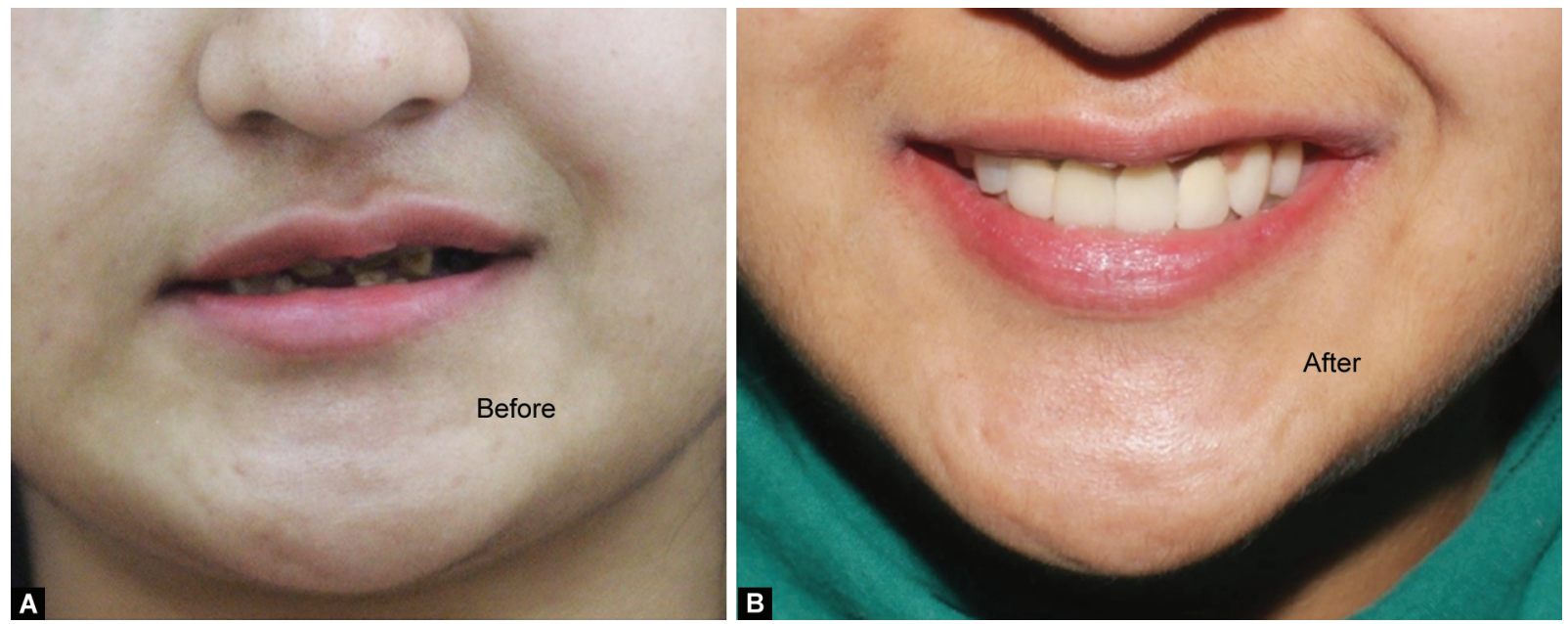

Figs $6 \mathrm{~A}$ and $\mathrm{B}$ : Comparison of pre- and post-treatment-smile view

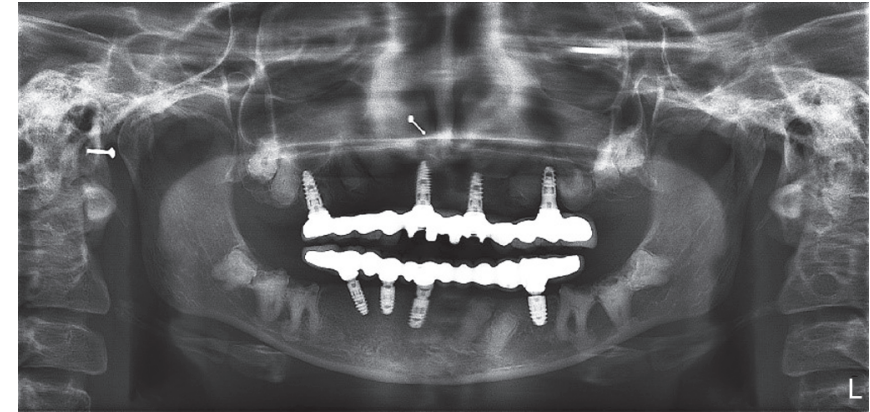

Fig. 7: One-year follow-up post-cementation

biotype during screwing and unscrewing of the suprastructure. This thereby would maintain the peri-implant health more efficiently. ${ }^{17}$ The patient had a constricted ridge and implants were placed to accommodate the native topography; thus, angulated abutments were chosen accordingly. A hybrid prosthesis called "Malo Bridge" was designed on screw-retained multiunit abutments to hide the unesthetic buccal screw access holes to compensate for the variations in the angulation.

\section{Conclusion}

A multidisciplinary (periodontics, orthodontics, and surgery) is mandated for a successful treatment outcome for cases like this. Also, conservative treatment decisions have to be made to deliver patient comfort, function, and esthetic without compromising the native biology. Therefore, in young patients like these we can hope to provide them with a prosthesis with a more predictable outcome, with multiunit abutments and a hybrid prosthesis.

\section{References}

1. Bayar GR, Ortakoglu K, Sencimen M. Multiple impacted teeth: report of 3 cases. Eur J Dent 2008;2(1):73-78. DOI: 10.1055/s-0039-1697358.

2. Sujatha G, Sivapathasundharam B, Sivakumar G, et al. Idiopathic multiple impacted unerupted teeth: case report and discussion. J Oral Maxillofac Pathol 2012;16(1):125-127. DOI: 10.4103/0973-029X.92989.

3. Carey JC. Syndromes of the head and neck Gorlin RJ, Michael Cohen M, Stefan Levin L, ed., New York: Oxford University Press; 1990. p. 977. Am J Med Genet 1992;42:144-144.
4. Guruprasad Y, Naik RG. Multiple impacted teeth in a non-syndromic patient. SRM J Res Dent Sci 2012;3(4):279-280. DOI: 10.4103/0976433X.114972.

5. Saluja H, Mahindra U, Gaikwad PT, et al. Multiple impacted teeth in maxilla in a young non syndromic patient: a rare occurrence: a case report with review of literature. Pravara Med Rev 2012;4:23-24.

6. Saluja KS, Singh B, Bhatia TK. An atypical case of non-syndromic multiple impacted supernumerary teeth - a case report. Int J Contemp Med Res 2016;3:1423-1425.

7. Ghaeminia $\mathrm{H}$, Nienhuijs MEL, Toedtling $\mathrm{V}$, et al. Surgical removal versus retention for the management of asymptomatic diseasefree impacted wisdom teeth. Cochrane Database Systema Rev 2020(5):CD003879.

8. Resnik RR, Misch C. Prophylactic antibiotic regimens in oral implantology: rationale and protocol. Implant Dent 2008;17(2):142150. DOI: 10.1097/ID.0b013e3181752b09.

9. Taruna M, Chittaranjan B, Sudheer N, et al. Prosthodontic perspective to all-on- $4^{\circledast}$ concept for dental implants. J Clin Diagn Res 2014;8(10):ZE16-ZE19. DOI: 10.7860/JCDR/2014/9648.5020.

10. Branemark PI, Svensson B, van Steenberghe D. Ten year survival rates of fixed prostheses on four or six implants ad modum Branemark in full edentulism. Clin Oral Implants Res 1995;6(4):227-231. DOI: 10.1034/j.1600-0501.1995.060405.x.

11. Ashurko I, Trofimov A, Tarasenko S, et al. Full-mouth screw-retained implant-supported rehabilitation with multiunit abutments using virtual guided surgery and digital prosthetics protocol. Case Rep Dent 2020;2020:3585169. DOI: 10.1155/2020/3585169.

12. Gulnar A, Altintas SH, Yilmaz O, et al. Rehabilitation of the completely edentulous young patient with the "Malo Bridge": a case report. Niger J Clin Pract 2020;23(9):1328-1331. DOI: 10.4103/njcp.njcp_170_20.

13. Kodama T. Implant-supported full-mouth reconstruction Malo implant bridge. J Calif Dent Assoc 2012;40(6):497-508.

14. Sharma A. A rare non-syndrome case of concomitant multiple supernumerary teeth and partial anodontia. J Clin Pediatr Dent 2001;25(2):167-169. DOI: 10.17796/jcpd.25.2.k4617k5126205k46.

15. Yildirim D, Yilmaz HH, Aydin U. Multiple impacted permanent and deciduous teeth. Dentomaxillo Radiol 2004;33(2):133-135. DOI: $10.1259 / \mathrm{dmfr} / 12620936$.

16. Sivakumar A, Valiathan A, Gandhi S, et al. Idiopathic failure of eruption of multiple permanent teeth: report of 2 adults with a highlight on molecular biology. Am J Orthod Dentofacial Orthop 2007;132(5):687692. DOI: 10.1016/j.ajodo.2006.04.034.

17. Chai WL, Razali M, Ngeow MC. Dimension and structures of biological seal of peri-implant tissues. In Dental implantology and biomaterial, ch. 3, London, UK: IntechOpen; 2016. 